\title{
ARTICLE \\ Receptor and circuit mechanisms underlying differential procognitive actions of psychostimulants
}

\author{
Robert C. Spencer ${ }^{1}$ and Craig W. Berridge ${ }^{1}$
}

\begin{abstract}
Psychostimulants, including methylphenidate (MPH), improve cognitive processes dependent on the prefrontal cortex (PFC) and extended frontostriatal circuitry. In both humans and animals, systemic MPH improves certain cognitive processes, such as working memory, in a narrow inverted-U-shaped manner. In contrast, other processes, including attention-related, are improved over a broader/right-shifted dose range. The current studies sought to elucidate the potential circuit and receptor mechanisms underlying the divergent dose-dependent procognitive effects of psychostimulants. We first observed that, as with working memory, although sustained attention testing was highly dependent on multiple frontostriatal regions, only MPH infusion into the dorsomedial PFC improved task performance. Importantly, the dose-response curve for this action was right-shifted relative to working memory, as seen with systemic administration. Additional studies examined the receptor mechanisms within the PFC associated with the procognitive actions of MPH across working memory and sustained attention tasks. We observed that PFC a2 and D1 receptors contributed to the beneficial effects of MPH across both cognitive tasks. However, a1 receptors only contributed to MPH-induced improvement in sustained attention. Moreover, activation of PFC a1 receptors was sufficient to improve sustained attention. This latter action contrasts with the impairing actions of PFC a1 receptors reported previously for working memory. These results provide further evidence for a prominent role of the PFC in the procognitive actions of MPH and demonstrate the divergent dose sensitivity across cognitive processes aligns with the differential involvement of PFC a1 receptors.
\end{abstract}

Neuropsychopharmacology (2019) 44:1820-1827; https://doi.org/10.1038/s41386-019-0314-y

\section{INTRODUCTION}

At low and clinically relevant doses, psychostimulants, including methylphenidate (MPH, Ritalin), enhance cognition dependent on the prefrontal cortex (PFC) and extended frontostriatal circuitry [1-3]. These actions are observed in individuals with attention deficit hyperactivity disorder (ADHD) as well as healthy human and animal subjects [4-7]. Converging observations indicate that drug action within the PFC contributes to the procognitive effects of psychostimulants (for review see [1]). This includes the fact that direct infusion of MPH and other ADHD-approved treatments into the PFC of rats and monkeys improves PFC-dependent cognition as measured in tests of working memory [8-10].

Psychostimulants have been typically viewed to uniformly affect a diversity of PFC-dependent cognitive processes [11, 12]. However, increasing evidence in humans and animals demonstrates that subsets of PFC-dependent cognitive processes vary in their sensitivity to these drugs. Specifically, working memory and response inhibition display relatively narrow inverted-U dosedependent facilitation, while overt behavior and different forms of attention are improved across a broader and right-shifted range of doses $[6,13,14]$. To date, the neural mechanisms responsible for the differential sensitivity of PFC-dependent cognitive processes to psychostimulants are unclear, representing a critical weakness in our understanding of this clinically important class of drugs.

The PFC extends topographically-organized projections to the striatum, forming functional frontostriatal circuits $[15,16]$. In rodents, the dorsomedial PFC (dmPFC), encompassing the dorsal anterior cingulate and dorsal prelimbic PFC, and dorsomedial striatum (dmSTR) are strongly implicated in higher cognitive function $[17,18]$. Consistent with this, prior studies demonstrate that, in rats, working memory performance is highly dependent on the dmPFC and the dmSTR as well as the ventromedial striatum (vmSTR; $[1,8,19])$. Relative to low and clinically relevant doses, higher doses of psychostimulants elicit larger and more uniform elevations in norepinephrine (NE) and dopamine (DA) throughout the brain $[5,19,20]$. Thus, differences in sensitivity to psychostimulants across cognitive processes may reflect broader actions of higher doses across frontostriatal circuitry. To test this, the current studies first examined the degree to which MPH acts within distinct PFC and striatal subfields to improve sustained attention, a form of focused attention previously demonstrated to display a right-shifted dose sensitivity to MPH [6]. Similar to that seen with working memory [8], we observed that while multiple frontostriatal regions support sustained attention, only $\mathrm{MPH}$ infusion into the dmPFC improved this cognitive process. Importantly, as with systemic administration, the dose dependency of this action was right-shifted relative to that seen previously with working memory [8]. Combined, these observations indicate that the differences in dose sensitivity across PFCdependent cognitive processes to $\mathrm{MPH}$ arise from mechanisms intrinsic to the PFC.

One such mechanism could involve differential actions of PFC catecholamine receptors across tasks. Within the PFC, catecholamines exert non-monotonic modulatory actions on cognition that

\footnotetext{
${ }^{1}$ Department of Psychology, University of Wisconsin-Madison, Madison, WI 53706, USA
}

Correspondence: Robert C. Spencer (rcspencer@wisc.edu)

Received: 4 September 2018 Revised: 3 January 2019 Accepted: 5 January 2019

Published online: 11 January 2019 
are both receptor subtype- and cognitive process-dependent. Thus, in the case of working memory, PFC DA D1 receptors elicit an inverted-U-shaped modulatory action, with both low and high rates of activation associated with impairment [21, 22]. PFC NE also exerts an inverted- $U$ modulation of working memory. However, in this case the beneficial actions are mediated by high-affinity a 2 receptors, while the impairing actions of high rates of NE release involve activation of lower affinity a1 receptors [23]. Consistent with this, the working memory-enhancing actions of $\mathrm{MPH}$ are prevented by systemically administered $\mathrm{a} 2$ and D1 antagonists [3]. In contrast to that seen with working memory, activation of PFC a1 receptors improves flexible attention as measured in an attention set shifting task [24]. Based on these and other observations, it was initially proposed that high rates of NE release (e.g. stress) in the PFC promote flexible attention at the expense of focused attention via a1 receptor activation [24-26]. However, subsequent observations indicated that both flexible and focused attention display similar right-shifted dose sensitivities to MPH [6]. Moreover, MPH-induced improvement in focused attention is prevented by systemic administration of an a1 antagonist [6].

Collectively, these observations suggest that the procognitive actions of higher doses of MPH involve PFC a1 receptors. To test this, and to better understand PFC catecholamine receptor mechanisms regulating focused attention, additional studies examined the degree to which intra-PFC MPH-induced improvement in working memory and sustained attention is dependent on local a1, as well as $a 2$ and D1, receptors. We observed that PFC a1 receptors are necessary for $\mathrm{MPH}$-induced improvement in sustained attention, but not working memory. Moreover, activation of PFC a1 receptors was sufficient to improve sustained (focused) attention, identical to that seen previously for flexible attention [24]. In contrast to the task-selective involvement of PFC a1 receptors, both $a 2$ and D1 receptors in the PFC are necessary for the procognitive effects of MPH in both tasks.

These studies demonstrate a central role of the PFC in the procognitive actions of psychostimulants beyond those assessed in tests of working memory. Additionally, these studies further our understanding of the receptor mechanisms underlying the diverse procognitive actions of this widely used class of drugs as well as the neurobiology of PFC-dependent cognition.

\section{METHODS AND MATERIALS}

Animals

Male Sprague-Dawley rats (260-280 g; Charles River, Wilmington, MA) were pair-housed in polycarbonate cages on a 13-11 h light/ dark cycle (lights on 06:00). Animals were fed ad libitum for 7 days and subsequently restricted to $15-17 \mathrm{~g}$ of food/day available at 16:00. Testing/training was conducted between 09:00 and 16:00 $\mathrm{h}$ at the same time each day, $\pm 1 \mathrm{~h}$. Animals were weighed twice weekly and handled extensively prior to testing. All facilities and procedures were in accordance with National Institutes of Health (USA) guidelines and approved by the Institutional Animal Care and Use Committee.

\section{Surgery}

Following training (see below), rats were anesthetized with isoflurane. Twenty-five ga. stainless steel cannulae were stereotaxically (flat skull) implanted bilaterally over the dmPFC (i.e. dorsal anterior cingulate/dorsal prelimbic PFC; $\mathrm{A}+3.0 ; \mathrm{L} \pm 0.8$; $\mathrm{V}-0.2 \mathrm{~mm}$ below dura), vmPFC (ventral prelimbic/infralimbic PFC; same coordinates, longer needles), dmSTR ( $A+0.45 ; L \pm 2.0$; $\mathrm{V}-3.2 \mathrm{~mm})$, or vmSTR $(A+1.6 ; \mathrm{L} \pm 1.5 ; \mathrm{V}-3.6 \mathrm{~mm})$ and secured with stainless steel screws and acrylic cement (Plastics One, Roanoke, VA). Stainless steel stylets prevented occlusion of the cannulae.
Sustained attention

Animals were trained and tested in an operant-based signal detection test of sustained attention, as previously described $[5,6]$. Briefly, on half of 100 discrete trials (selected at random, $p=0.5$ ) an LED was illuminated and two levers were projected into the chamber ("signal trials"). The signal length was variable, randomly selected from the following list: $0.125,0.25,0.375,0.5,0.625,0.75$, $0.875,1.0 \mathrm{~s}$, with replacement. On the other half of trials, no signal occurred, after which both levers were inserted ("no-signal trials"). Levers remained in the chamber until a response was made, at which time they were retracted. On signal trials, a right lever press was scored as a "hit" and reinforced with sucrose ( $45 \mathrm{mg}$; Bio-Serv, Frenchtown, New Jersey) and a left lever press was scored as a "miss." On a no-signal trial, a right lever press was scored as a "false alarm" and a left lever press was reinforced ("correct rejection"). For correct response trials, houselights were illuminated for $5 \mathrm{~s}$. For incorrect trials, levers were retracted followed by a 5 -s time-out (houselights off). Failure to respond within $5 \mathrm{~s}$ of lever insertion triggered lever retraction and a 5-s time-out. There was a variable intertrial interval of $13 \mathrm{~s}$ on average (minimum $5 \mathrm{~s}$ ). Trials with no response occurred infrequently and were excluded from analyses. Animals were trained until performance reached $70-85 \%$. Dependent measures included the proportion of trials with a correct response (proportion of hits + proportion of correct rejection), probability of a hit (correct responses/number of signal trials), probability of a false alarm (correct responses/number of no-signal trials), and $d^{\prime}$, a relative measure of stimulus detection ability. $d^{\prime}=Z(N)-Z(S)$. $Z(N)=Z$ score of the noise distribution $=Z$ score of $(1$-probability of false alarms). $Z(S)=Z$ score of the signal $=Z$ score of (1-probability of a hit) [27].

Sustained attention testing involved the use of several separate cohorts with treatments spread among the different cohorts. Due to variations in performance between cohorts, all treatment effects were measured against vehicle treatment. In most cohorts treatments were replicated within animals.

\section{Working memory}

Prior to surgery, animals were trained in a T-maze (opaque black Plexiglas) with 10 trials/session, as described previously [28, 29]. Animals were rewarded ( $58 \mathrm{mg}$ chocolate chip or $45 \mathrm{mg}$ sucrose pellet) when they entered the maze arm not chosen on the previous trial. Between trials the animal was placed in a start box at the base of the maze with removable Plexiglas gate, with a zero-delay. Spatial cues were minimized with black plastic sheeting. Animal waste was removed by a dry tissue between trials and cleaned with $50 \%$ ethanol between animals. Treatments were counter-balanced within and across animals.

Following surgery, rats were retrained with 20 trials/session at zero delay until pre-surgery levels. Delay intervals were then introduced that resulted in 65-80\% accuracy $(5-120 \mathrm{~s}$; mean $=42$ $\pm 36 \mathrm{~s}$ ). Stable pre-treatment performance (baseline) was defined as two consecutive days in which performance did not differ greater than $10 \%$. In order to ensure stable performance, rats were run at the same delay for 2 days following treatment. Data were included in the analyses only when baseline and post-treatment performance did not differ by more than $10 \%$.

\section{Drug infusion}

MPH HCl $(0.03 \mu \mathrm{g}, 0.125 \mu \mathrm{g}, 0.5 \mu \mathrm{g}, 2.0 \mu \mathrm{g} / 500 \mathrm{nl})$, atimpamezole $(1.25 \mu \mathrm{g}$ or $0.625 \mu \mathrm{g} / 500 \mathrm{nl}), \mathrm{SCH} 23390(0.5 \mu \mathrm{g} / 500 \mathrm{nl})$, benoxathian $(0.4 \mu \mathrm{g} / 500 \mathrm{nl})$, or muscimol $(75 \mathrm{ng} / 500 \mathrm{nl})$ were obtained from Sigma-Aldrich (St. Louis, MO), and dissolved in buffered artificial extracellular fluid $\left(147 \mathrm{mmol} \mathrm{NaCl}, 1.3 \mathrm{mmol} \mathrm{CaCl}_{2}, 0.9\right.$ $\mathrm{mmol} \mathrm{MgCl}, 2.5 \mathrm{mmol} \mathrm{KCl} ; \mathrm{pH}=7.4)$. Bilateral $500 \mathrm{nl}$ infusions of drug/vehicle were made at a rate of $250 \mathrm{nl} / \mathrm{min}$ using $33 \mathrm{ga}$. needles that projected below the guide cannulae $(1.6-2.0 \mathrm{~mm}$, dmPFC; $4 \mathrm{~mm}$, vmPFC; $2.5 \mathrm{~mm}$, dmSTR; $4 \mathrm{~mm}$ vmSTR). Needles remained in tissue for 2 min following infusions, after which stylets 
were replaced. Prior to the first treatment, rats received two mock infusions, consisting of an initial needle insertion and $48 \mathrm{hs}$ later a vehicle infusion. Rats were placed in their home cage for $15 \mathrm{~min}$ following infusions. All treatments were separated by at least 2 days. To maintain tissue integrity and minimize infection, the number of treatments was limited to 10 per animal.

Histological analyses and data selection

Following testing, rats were deeply anesthetized and transcardially perfused with $3.7 \% \mathrm{w} / \mathrm{v}$ formaldehyde. Brains were stored in formaldehyde for at least $24 \mathrm{~h}$. Injector placement was verified in $40-\mu m$-thick Neutral Red-stained coronal sections. Data from a given experiment were included only when histological analyses verified accurate placement of injectors and a minimum of tissue damage.

\section{Statistical analyses}

Given limits on the number of infusions, as well as limits on working memory delay length, it was not possible for all animals to receive all doses. Therefore, results were analyzed using mixed linear models in JMP Pro 12 with treatment as a fixed effect and subjects as a random effect. Sustained attention testing was run with several separate cohorts. Because there are modest variations in performance within and between cohorts, all sustained attention treatment effects were calculated relative to vehicle in a within-subjects design. In the vast majority of cases, treatments were replicated in the same animal. Muscimol effects were modeled with region (dmPFC, dmSTR, vmSTR), treatment (vehicle, muscimol), and treatment $\times$ region as fixed effects and planned comparisons were corrected for multiple comparisons with Holm-Bonferroni correction. To assess the regional and dosedependent actions of MPH on sustained attention, we used separate models for each region. Two additional models were used for the sustained attention and working memory antagonist studies. For all models, subjects were treated as a random variable. Outside of muscimol testing, all planned comparisons to vehicle treatment were conducted using Dunnett's $t$-tests.

\section{RESULTS}

Frontostriatal circuitry associated with $\mathrm{MPH}$-induced improvement in sustained attention

Dependency of sustained attention on frontostriatal circuitry. In contrast to working memory, the frontostriatal neurocircuitry supporting sustained attention is poorly understood. Thus, we first examined the degree to which sustained attention is dependent on different frontostriatal regions using reversible inactivation via microinfusion of the GABA agonist, muscimol. Muscimol had a significant effect on performance $\left(F_{1,28.9}=86.0, p<0.0001\right)$. Inactivation of the $\operatorname{dmPFC}(n=10$; vehicle $n=10)$ and dmSTR ( $n=7$; vehicle $n=7$ ) reduced performance to chance levels, as assessed by a change in $d^{\prime}$ from vehicle, as well as other measures (Fig. 1; Table 1; dmPFC, $t_{29}=5.46 ; p<0.0001 ; d^{\prime}, d m S T R, t_{29}=$ $-3.19 ; p=0.04)$. Inactivation of the $\operatorname{vmSTR}(n=8$; vehicle $n=8)$ produced a larger impairment in sustained attention $\left(t_{29}=7.74\right.$; $p<0.0001$; Figure 1; Table I). However, in contrast to that seen with dmPFC or dmSTR inactivation, this reflected the fact that animals stopped responding in the presence of errors, potentially reflecting diminished motivation to engage in the task, rather than cognitive impairment per se.

Intra-PFC MPH infusions. MPH acts in the dmPFC, but not the vmPFC, to improve working memory in an inverted-U manner [28, 29]. To test whether MPH acts directly in the dmPFC or vmPFC to modulate sustained attention, animals received infusions of vehicle or varying doses of MPH into the either region. As shown in Fig. 2 and Table S2, infusion of MPH $(500 \mathrm{nl} ; 0.03 \mu \mathrm{g} n=6 ; 0.125$ $\mu \mathrm{g} n=8 ; 0.5 \mu \mathrm{g}, n=6 ; 2.0 \mu \mathrm{g}, n=13$; vehicle, $n=21$, due to multiple cohorts) into the dmPFC elicited an inverted-U-shaped improvement in sustained attention as measured by $d^{\prime}\left(F_{4,66.8}=\right.$ $4.15, p<0.01)$, with maximal improvement observed at the $0.5 \mu \mathrm{g} /$ hemisphere dose $(p=0.02)$ relative to vehicle. As observed with systemic administration (see Supplemental Figure S1), this inverted-U dose-response curve is right-shifted relative to that seen previously with intra-PFC MPH-induced improvements in working memory [8]. MPH infusion into the vmPFC had no significant effects on $d^{\prime}\left(\mathrm{F}_{4,24}=1.81, p=0.16\right.$; see Fig. 3; Supplemental Table S3; $0.03 \mu \mathrm{g}, n=7, p=1.0 ; 0.125 \mu \mathrm{g}, n=7, p=0.98$; $0.5 \mu \mathrm{g}, n=7, p=1.0 ; 2.0 \mu \mathrm{g}, n=7, p=0.10$; vehicle, $n=7)$. The one exception to this was a trend for improvement with the highest

\section{A Muscimol Inactivation}

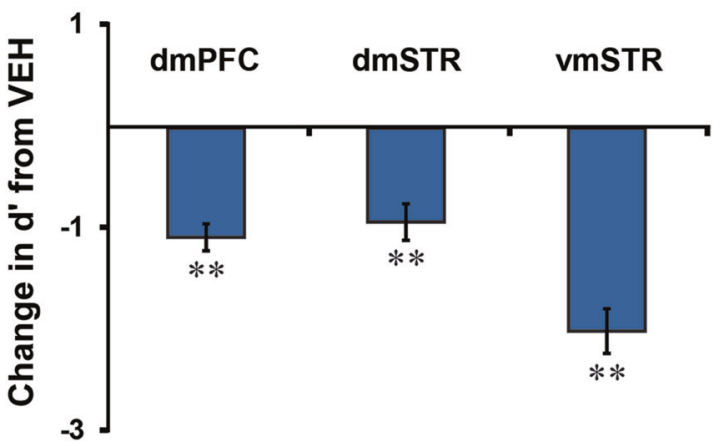

Fig. 1 Frontostriatal circuit mechanisms supporting sustained attention. Shown are the effects of muscimol ( $75 \mathrm{ng} / \mathrm{hemisphere)}$ inactivation of the dmPFC (dorsal anterior cingulate/dorsal prelimbic PFC), dmSTR, or vmSTR on performance in a sustained attention task. Bars represent mean change in $d^{\prime}$ from vehicle treatment \pm SEM. Performance was impaired, as measured by a change in $d^{\prime}$ sensitivity compared to vehicle, with inactivation of all three regions. For the dmPFC and dmSTR, this resulted in nearly chance levels of performance (see Table I). In contrast, vmSTR inactivation produced a more profound impairment that largely reflects the fact that animals ceased performing the task following a series of errors. ${ }^{*} p<0.05,{ }^{*} p<0.01$ compared to vehicle treatment

Table 1. Muscimol $(75 \mathrm{ng} / \mathrm{hemisphere})$ inactivation within frontostriatal nodes

\begin{tabular}{|c|c|c|c|c|c|}
\hline Region & $d^{\prime}$ & Prop correct & $\operatorname{Pr}$ (hit) & $\operatorname{Pr}(\mathrm{FA})$ & No responses \\
\hline dmPFC & $1.07 \pm 0.20^{* *}$ & $0.18 \pm 0.04^{* *}$ & $-0.24 \pm 0.06^{* *}$ & $0.12 \pm 0.05^{*}$ & $-1.07 \pm 3.39$ \\
\hline dmSTR & $0.88 \pm 0.28^{*}$ & $-0.12 \pm 0.05$ & $-0.18 \pm 0.08^{* *}$ & $0.11 \pm 0.07$ & $1.14 \pm 4.96$ \\
\hline vmSTR & $1.99 \pm 0.26^{* *}$ & $0.55 \pm 0.05^{* *}$ & $-0.30 \pm 0.07 * *$ & $0.32 \pm 0.06 * *$ & $44.19 \pm 4.64^{* *}$ \\
\hline
\end{tabular}


A Sustained Attention
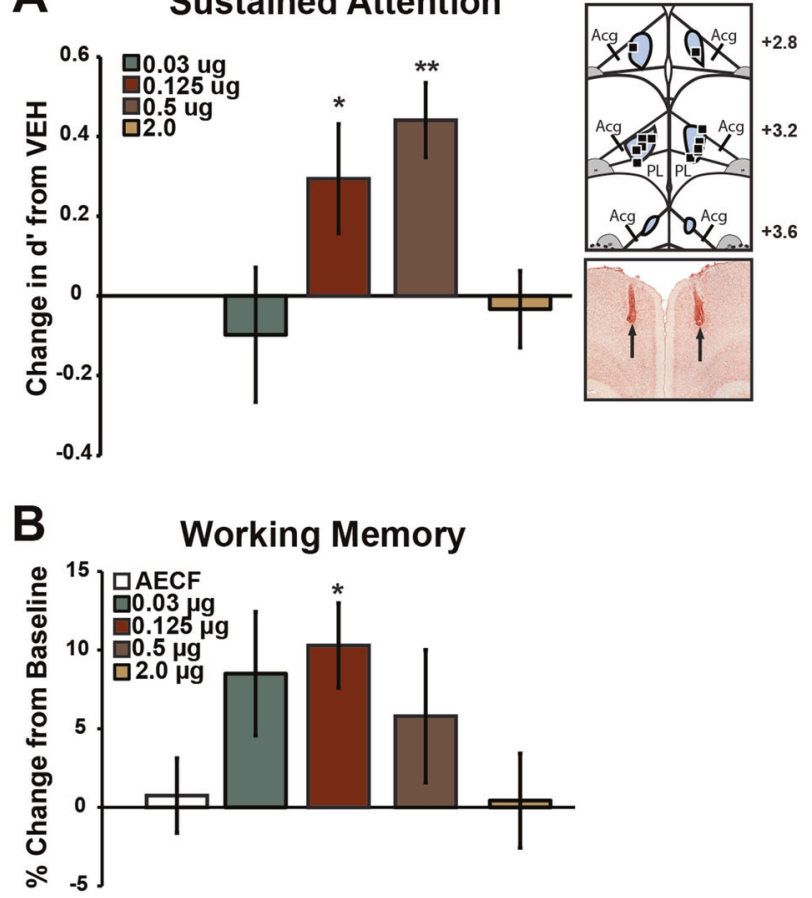

Fig. $2 \mathrm{MPH}$ acts in the dmPFC to improve both sustained attention and working memory. a Left: Infusion of MPH into the dmPFC (dorsal anterior cingulate/dorsal prelimbic PFC) improves sustained attention in an inverted-U-shaped manner, with $0.5 \mu \mathrm{g}$ producing maximal improvement as measured by a change in $d^{\prime}$ sensitivity from vehicle (mean \pm SEM). Top Right: Schematic of all infusion sites for the $0.5 \mu \mathrm{g}$ dose. Numbers represent anterior/posterior coordinates of coronal sections. Bottom Right: Representative photomicrograph of MPH infusion site in the dmPFC showing the main body of the needle track. Arrows indicate the ventral most location of the track within the dmPFC. Cannulae are only lowered into the dmPFC $\sim 200 \mu \mathrm{m}$, minimizing damage. b Previously published data regarding the dose-response curve for $\mathrm{MPH}$-induced improvement in working memory [8]. When compared with data presented in panel a, it is clear that sustained attention displays right-shifted dose sensitivity relative to working memory. Acg dorsal anterior cingulate, PL prelimbic PFC. ${ }^{*} p<0.05,{ }^{* *} p<0.01$ compared to vehicle treatment

dose of $\mathrm{MPH}$, likely reflecting diffusion of the drug into the dmPFC at a lower concentration.

Intra-striatal MPH infusions. Additional studies examined the sustained attention effects of MPH within the dmSTR and vmSTR. As shown in Fig. 3 , similar to that observed previously for working memory [8], neither MPH infusion into the dmSTR nor vmSTR had a significant effect on sustained attention as measured by a change in $d^{\prime}$ from vehicle treatment (see also Supplemental Table S3; dmSTR: $F_{4,68.3}=1.32, p=0.27 ; 0.03 \mu g, n=7, p=1.0$; $0.125 \mu \mathrm{g}, n=12, p=0.71 ; 0.5 \mu \mathrm{g}, n=11, p=0.87 ; 2.0 \mu \mathrm{g}, n=12$, $p=0.35$; vehicle, $n=29$, due to multiple cohorts; vmSTR: $F_{3,20.3}=$ $0.13, p=0.94 ; 0.125 \mu \mathrm{g}, n=8, p=0.93 ; 0.5 \mu \mathrm{g}, n=8, p=0.99$; $2.0 \mu \mathrm{g}, n=8, p=0.90$; vehicle $n=8$ ).

Receptor mechanisms underlying beneficial actions of MPH on working memory vs. sustained attention

Additional studies examined if the difference in dose sensitivity across tasks reflects differences in PFC catecholamine receptor mechanisms. Animals received either intra-dmPFC infusions of vehicle, $\mathrm{MPH}$, the a1 antagonist, benoxathian, a2 antagonist, atipamezole, or the D1 antagonist, SCH23390, either alone or with $\mathrm{MPH}$. Animals were then tested in our working memory or sustained attention tasks. The dose of MPH used was the maximally-improving dose for each task (see Fig. 2, [6]). Doses of the antagonists were based on prior published work [10, 30, 31] as well as extensive pilot studies that identified doses subthreshold for altering performance (data not shown).

Working memory. Animals received intra-PFC infusions of vehicle $(n=13), 0.125 \mu \mathrm{gPH}(n=11), \mathrm{MPH}+\mathrm{a} 1$ antagonist $(0.4 \mu \mathrm{g}, n=$ $11), \mathrm{MPH}+\mathrm{a} 2$ antagonist $(1.25 \mu \mathrm{g}, n=10)$, or $\mathrm{MPH}+\mathrm{D} 1$ antagonist $(0.5 \mu \mathrm{g}, n=9)$. As shown in Fig. 4 , there was an overall effect of treatment $\left(F_{85}=2.3, p=0.03\right)$ with $\mathrm{MPH}$ infusion into the dmPFC significantly improving performance relative to vehicle $\left(t_{85}=2.99, p=0.02\right)$. When infused into the dmPFC alone, neither the a1 antagonist $\left(n=9, t_{85}=0.97, p=0.89\right)$, a 2 antagonist $(n=$ $\left.10, t_{85}=0.75, p=0.97\right)$ nor the D1 antagonist $\left(n=12, t_{85}=0.33\right.$, $p=0.97)$ significantly affected performance. When co-infused with $\mathrm{MPH}$, at a dose previously shown to block NE-dependent improvement in attention set shifting [24], the a1 antagonist had no effect on MPH-induced improvement in working memory $\left(t_{85}=2.75, p=0.04\right)$. In contrast, the $a 2$ antagonist $\left(t_{85}=0.73, p=\right.$ $0.97)$ and the D1 antagonist $\left(t_{85}=0.73, p=0.97\right)$ completely blocked the cognition-enhancing actions of intra-PFC MPH.

Sustained attention. Animals received intra-PFC infusions of vehicle $(n=45), 0.5 \mu \mathrm{gPH}(n=21), \mathrm{MPH}+\mathrm{a} 1$ antagonist $(0.5$ $\mu \mathrm{g}, n=16), \mathrm{MPH}+\mathrm{a} 2$ antagonist $(0.625 \mu \mathrm{g}, n=16)$, or MPH + D1 antagonist $(0.5 \mu \mathrm{g}, n=15)$. As shown in Fig. 4 and Supplemental Table S3, there was an overall effect of treatment $\left(F_{282.3}=3.87\right.$, $p=0.0002$ ), with infusion of $0.5 \mu \mathrm{g} \mathrm{MPH}$ into the dmPFC significantly improving performance relative to vehicle $\left(t_{282.3}=\right.$ $2.9, p=0.03)$. When infused into the dmPFC alone, no significant effects on performance were observed for any of the antagonists $\left(\mathrm{a} 1, n=20, t_{282.3}=-0.22, p=1.0 ; \mathrm{a} 2, n=14, t_{282.3}=0.93, p=\right.$ 0.96 ; $\left.\mathrm{D} 1, n=20, t_{282.3}=-0.86, p=0.98\right)$. In contrast to that seen with working memory, a1 receptor blockade prevented the sustained attention improving action of $\mathrm{MPH}\left(t_{282.3}=1.42, p=\right.$ 0.72). Similar to that seen with working memory, $\mathrm{MPH}$-induced improvement in sustained attention was prevented by dmPFC a2 $\left(t_{282.3}=0.02, p=1.0\right)$ and D1 antagonists $\left(t_{282.3}=0.35, p=1.0\right)$.

a-1 receptor stimulation in the dmPFC improves sustained attention. These latter observations suggest that a1 receptors in the PFC facilitate sustained attention. To directly test this, we examined the effects of intra-PFC infusion of the a1 agonist, phenylephrine, on sustained attention at a dose previously shown to impair working memory [32]. As shown in Fig. 4 and Supplemental Table S4, activation of dmPFC a1 receptors $(n=$ 13) improved sustained attention relative to vehicle treatment as measured by $d^{\prime}\left(t_{282.3}=3.68, p=0.002\right)$, indistinguishable from that of $\mathrm{MPH}\left(t_{282.3}=-0.91, p=0.36\right)$.

\section{DISCUSSION}

Psychostimulants are the most effective and widely used treatment for ADHD, reversing core frontostriatal cognitive deficits associated with this disorder [7, 33, 34]. Prior observations demonstrate a subset of PFC-dependent processes display a narrow inverted-U dose sensitivity to MPH (e.g. working memory, response inhibition), while others display a rightshifted dose sensitivity (flexible attention, focused attention, behavioral calming) $[6,13,14,35]$. Prior to the current studies, the mechanisms responsible for this differential sensitivity of PFC-dependent cognitive processes to psychostimulants had been unexplored, representing in a critical gap in our understanding of this widely used class of drugs. To initially address this, the current studies examined whether differences in dose sensitivity to $\mathrm{MPH}$ across tests of working memory vs. 


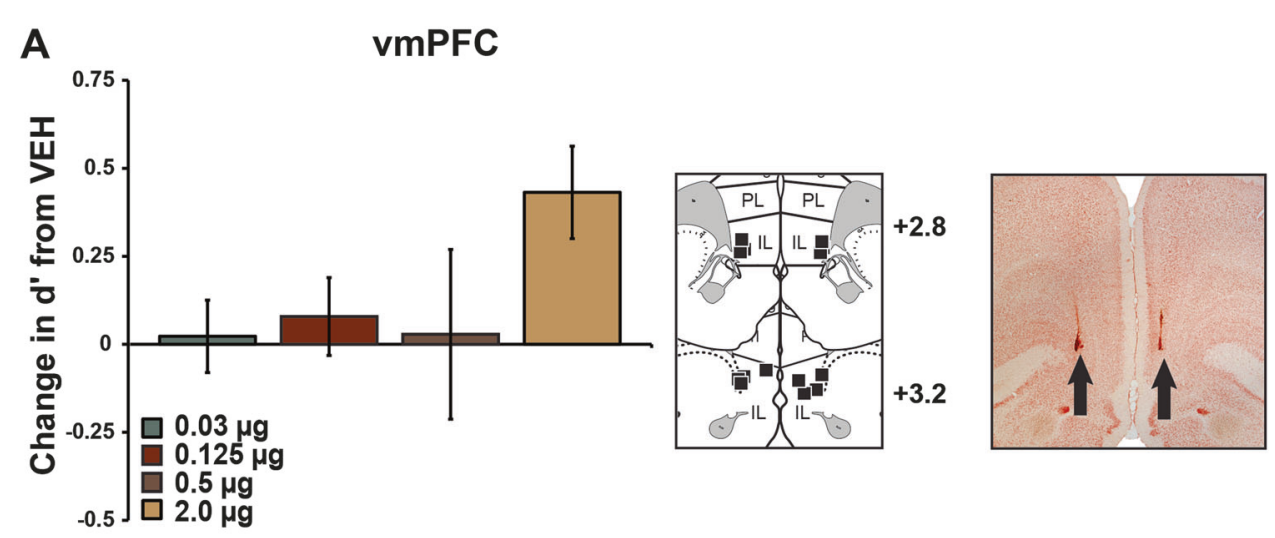

B
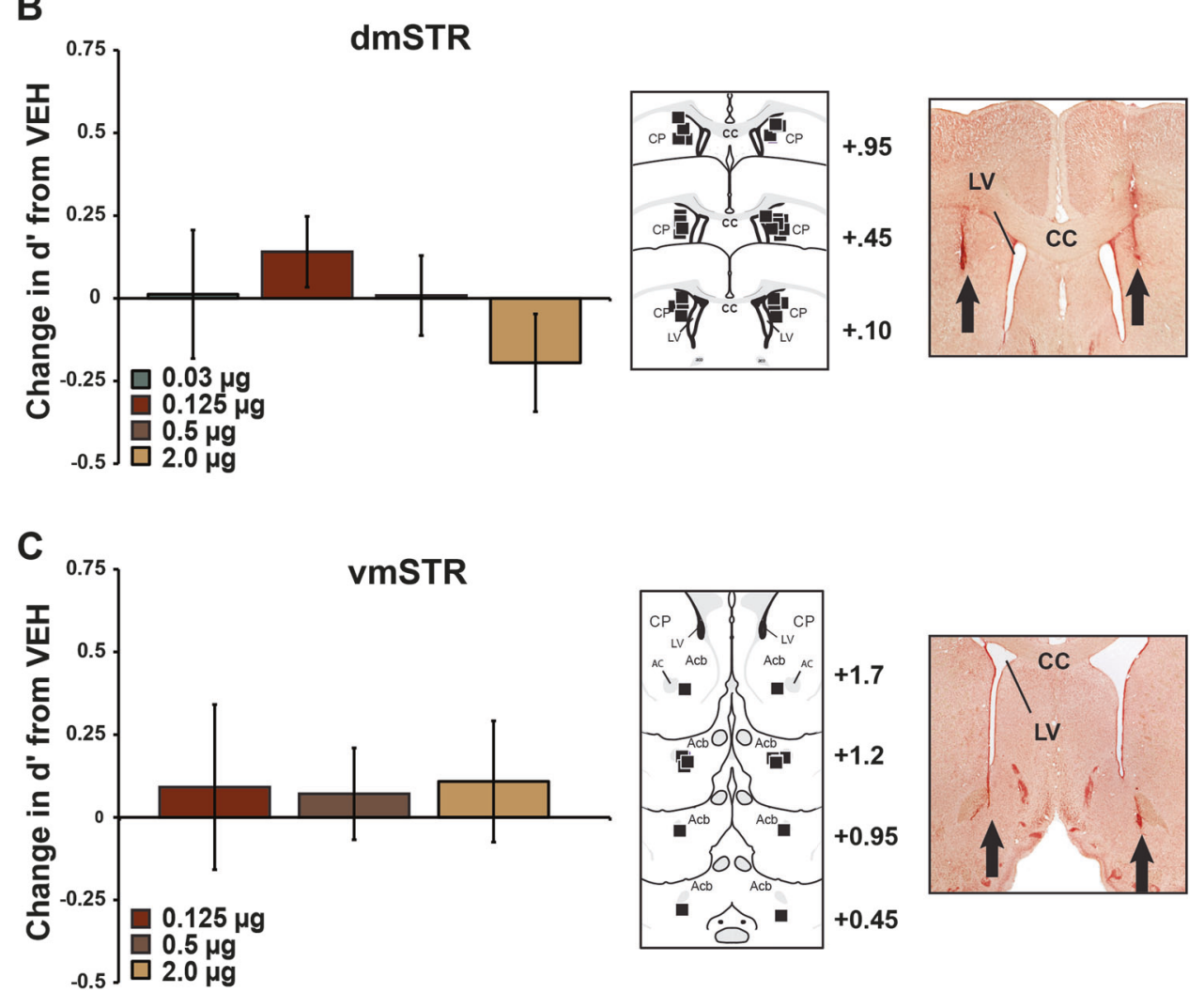

Fig. $3 \mathrm{MPH}$ actions outside the dmPFC. Infusion of MPH into the vmPFC (a), dmSTR (b), or vmSTR (c) had no effect on sustained attention as measured by change in $d^{\prime}$ compared with vehicle treatment (mean $\left.\pm \mathrm{SEM}\right)$. A trend for improvement was seen with the highest dose $(2.0 \mu \mathrm{g})$ of MPH infused into the vmPFC (ventral prelimbic/infralimbic PFC), which likely reflects diffusion into the dmPFC at a lower concentration. Schematics depict all MPH infusion sites for each region. Numbers represent anterior/posterior coordinates of coronal sections. Photomicrographs depict representative infusion sites for each region. Arrows indicate ventral most extent of infusion needles. PL prelimbic PFC, IL infralimbic PFC, CP caudate putamen, CC corpus callosum, Acb nucleus accumbens, LV lateral ventricle

sustained attention arise from varying circuit and/or receptor mechanisms.

These studies provide the first demonstration that sustained attention is dependent on multiple frontostriatal regions, similar to that reported previously for working memory [8]. Nonetheless, as with working memory [8], MPH only improved sustained attention when infused into the dmPFC, and not the vmPFC, dmSTR, or vmSTR. Importantly, the dose-response curve for this action was right-shifted relative to that previously seen with intradmPFC MPH in working memory [8]. This difference in dose sensitivity across tasks is identical to that seen with systemic administration [6]. Thus, regardless of route of administration, sustained attention is maximally improved at a dose four-fold higher than the maximally working memory-improving dose.
Additionally, regardless of route of administration, at this higher dose, working memory is no longer improved (Fig. 2; Supplemental Figure $1 ;[6,8])$. These observations demonstrate the differential dose sensitivity of these cognitive processes to MPH involves, at least in part, mechanisms contained within the PFC.

Additional studies provided the first examination of the PFC receptor mechanisms underlying the procognitive actions of MPH across working memory and sustained attention. These studies demonstrate that $a 2$ and D1 receptors within the PFC contribute to the procognitive actions of $\mathrm{MPH}$ observed in both tasks. In contrast, PFC a1 receptors only contribute to $\mathrm{MPH}$-induced improvement in sustained attention. Consistent with this, activation of PFC a1 receptors was sufficient to improve sustained attention. This contrasts with the working memory impairing 

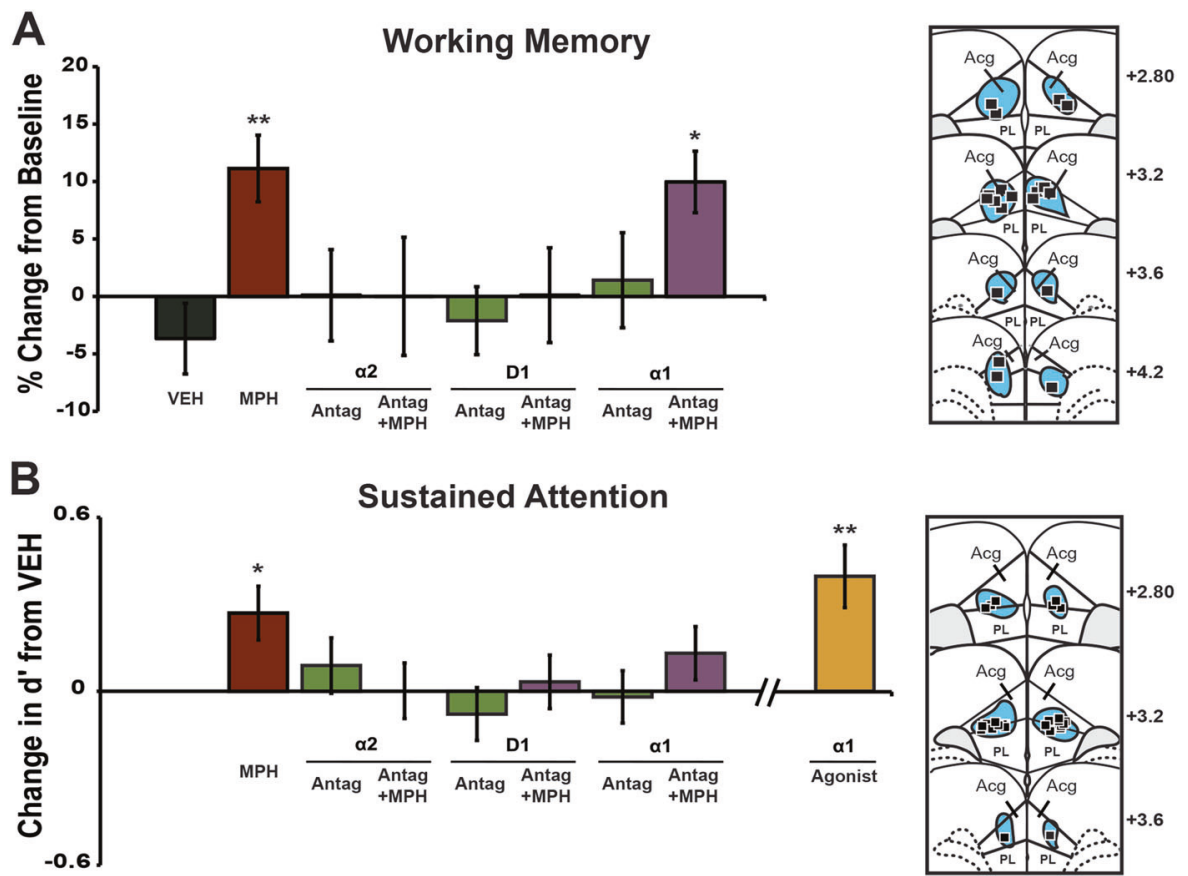

Fig. 4 PFC receptor mechanisms underlying the divergent dose-response curves of MPH across working memory and sustained attention. a The working memory-enhancing actions of intra-dmPFC (dorsal anterior cingulate/ dorsal prelimbic) MPH (0.125 $\mu \mathrm{g})$ are blocked with coinfusion of the $\alpha 2$ antagonist atipamezole $(1.25 \mu \mathrm{g})$ or the D1 antagonist SCH23390 $(0.5 \mu \mathrm{g})$, but not the $\alpha 1$ antagonist benoxathian $(0.4 \mu \mathrm{g})$ at doses that alone do not alter performance as measured by the percent change in performance from baseline. Schematic depicts all infusion sites with MPH (squares). b Similar to working memory, the sustained attention-enhancing actions of intra-dmPFC MPH (0.5 $\mu \mathrm{g})$ were blocked with co-infusion of the $\alpha 2$ antagonist atipamezole $(0.625 \mu \mathrm{g})$ or the D1 antagonist SCH23390 $(0.5 \mu \mathrm{g})$. However, in contrast to working memory, the $\alpha 1$ antagonist, benoxathian $(0.4 \mu \mathrm{g})$, also blocked intra-dmPFC MPH-induced improvements in sustained attention. Consistent with this latter observation, intra-dmPFC infusion of a dose of the $\alpha 1$ agonist, phenylephrine $(0.1 \mu \mathrm{g})$, previously shown to impair working memory $[32,36]$ significantly improved sustained attention, as measured by change in $d^{\prime}$ from vehicle (mean \pm SEM). The magnitude of improvement is comparable to that seen with intra-dmPFC MPH (0.5 $\mu \mathrm{g})$. Schematic depicts all infusion sites with MPH depicted (squares). Numbers represent anterior/posterior coordinates of coronal sections. Acg dorsal anterior cingulate, PL prelimbic PFC. ${ }^{*} p<0.05,{ }^{* *} p<0.01$ compared to vehicle or baseline

actions of PFC a1 receptors [32, 36]. Given a1 receptors display a lower affinity than 22 receptors, this could explain why sustained attention displays a right-shifted dose sensitivity relative to working memory. Collectively, these observations provide new insight into the neurobiology underlying both PFC-dependent cognition and the procognitive actions of psychostimulants used in the treatment of ADHD.

The PFC and procognitive actions of psychostimulants

The current study demonstrates that both sustained attention and working memory depend on multiple nodes of frontostriatal circuitry [8]. This is consistent with the fact that ADHD is associated with frontostriatal dysfunction [37-39] and that clinically relevant doses of MPH increase catecholamine signaling broadly within this circuit $[5,20]$. Nonetheless, only MPH action in the dmPFC, and not the vmPFC, dmSTR, or vmSTR, is sufficient to improve both sustained attention and working memory [8]. The preferential sensitivity of the dmPFC vs. the vmPFC in the procognitive actions of MPH is consistent with a well-known functional topographical organization of the rodent medial PFC, with dorsal regions more closely associated with "executive" cognitive processes $[40,41]$.

The two other ADHD-approved medications, a2 agonists and selective NE reuptake inhibitors, have been demonstrated to also act in the dorsal PFC of primates and rodents to improve working memory $[9,10,36,42]$. Combined, these observations demonstrate a prominent role of the PFC in the procognitive actions of all approved treatments for ADHD. Nonetheless, this does not rule out a role of the striatum in the therapeutic effects of psychostimulants. Given clinically relevant doses of psychostimulants elevate striatal DA neurotransmission $[5,20]$ it is possible that, when combined with drug action in the PFC, drug action in the striatum may contribute to greater efficacy of psychostimulants in ADHD relative to noradrenergic-selective treatments [43].

Receptor mechanisms within the PFC differentially contribute to the procognitive actions of $\mathrm{MPH}$

NE and DA act directly within the PFC to exert inverted-U-shaped modulation of working memory [44, 45]. For $N E$, this reflects procognitive actions of high-affinity postsynaptic $a_{2}$-receptors, engaged at lower rates of release, and working memory impairing actions of lower affinity $a_{1}$-receptors, engaged at higher rates of release (e.g. stress; [10, 32, 46]). For DA, moderate D1 receptor activation promotes, while higher rates of activation impair working memory performance [21]. Consistent with these observations, the current studies demonstrate that within the PFC, a2 and D1, but not a1, are necessary for $\mathrm{MPH}$-induced improvement in working memory. In contrast, MPH-induced improvement in sustained attention was dependent on all three receptor subtypes.

Prior studies demonstrate that activation of PFC a1 receptors promote a flexible form of attention as measured in an attention set shifting task [24]. Based on earlier observations, it was posited that PFC a1 receptors promote "flexible" attention at the expense of focused attention [25]. However, in our studies, activation of PFC a1 receptors improved sustained/focused attention, identical to flexible attention [24]. Collectively, these observations indicate that although a1 receptors differentially regulate distinct PFCdependent cognitive processes, this cannot be ascribed to a 
selective enhancement of attentional/cognitive "flexibility". One important difference between tests of working memory and tests of attention is the need in working memory tasks to actively maintain and protect from distractors information needed to make a subsequent action. Neurophysiologically, a2 and D1 receptors promote sustained activation of PFC neuronal activity during the delay interval of a working memory task, while a1 receptors degrade delay-related activity [47]. Thus, stimulation of PFC a2/D1 receptors help maintain internally generated representations. Future studies will need to determine if the proattentional actions of a1 receptors results from an increased sensitivity to external stimuli, as seen with more posterior cortical and subcortical areas [48] or more generally to increased attention to the environment.

\section{Clinical implications}

The neurocircuitry underlying the therapeutic actions of psychostimulants remains poorly understood. The current observations add to a growing body of evidence that the procognitive actions of ADHD-approved drugs involve (to some extent) direct action in the PFC $[8,23]$. This provides important information for future drug discovery targeted at the development of novel treatments for ADHD (e.g. [49]).

Sprague and Sleator [13] first described a differential dose sensitivity across varying cognitive/behavioral processes to MPH in children with ADHD, an observation later confirmed by Tannock et al. [14]. Our observations suggest an involvement of PFC a1 receptors in the procognitive actions of moderately higher doses of psychostimulants. It remains to be determined whether the facilitation of these a1-sensitive processes contribute to the beneficial (behavioral calming and attention improving) vs. detrimental (cognitive constriction) actions of psychostimulants and whether this differs across ADHD subtype [50]. Lastly, our results demonstrate a role of PFC a1 receptors in the regulation of focused attention, similar to that reported for flexible attention [24]. This may have relevance for the development of novel attention-enhancing compounds.

\section{FUNDING AND DISCLOSURE}

This work was supported by PHS grants, MH081843, MH098631, and the Office of the Vice Chancellor for Research and Graduate Education at the University of Wisconsin-Madison with funding from the Wisconsin Alumni Research Foundation. The authors declare no competing interests.

\section{ADDITIONAL INFORMATION}

Supplementary Information accompanies this paper at (https://doi.org/10.1038/ s41386-019-0314-y).

Publisher's note: Springer Nature remains neutral with regard to jurisdictional claims in published maps and institutional affiliations.

\section{REFERENCES}

1. Spencer R, Devilbiss D, Berridge C. The cognition-enhancing effects of psychostimulants involve direct action in the prefrontal cortex. Biol Psychiatry. 2015;77:940-50.

2. Mehta MA, Owen AM, Sahakian BJ, Mavaddat N, Pickard JD, Robbins TW. Methylphenidate enhances working memory by modulating discrete frontal and parietal lobe regions in the human brain. J Neurosci. 2000;20:RC65.

3. Arnsten AF, Dudley AG. Methylphenidate improves prefrontal cortical cognitive function through alpha2 adrenoceptor and dopamine D1 receptor actions: relevance to therapeutic effects in attention deficit hyperactivity disorder. Behav Brain Funct. 2005;1:2.

4. Mehta MA, Goodyer IM, Sahakian BJ. Methylphenidate improves working memory and set-shifting in AD/HD: relationships to baseline memory capacity. J Child Psychol Psychiatry. 2004;45:293-305.
5. Berridge CW, Devilbiss DM, Andrzejewski ME, Arnsten AF, Kelley AE, Schmeichel $B$, et al. Methylphenidate preferentially increases catecholamine neurotransmission within the prefrontal cortex at low doses that enhance cognitive function. Biol Psychiatry. 2006;60:1111-20.

6. Berridge C, Shumsky J, Andrzejewski M, McGaughy J, Spencer R, Devilbiss D, et al. Differential sensitivity to psychostimulants across prefrontal cognitive tasks: differential involvement of noradrenergic a(1)- and a(2)-receptors. Biol Psychiatry. 2012;71:467-73.

7. Mehta MA, Sahakian BJ, Robbins TW. Comparative psycholpharmacology of methylphenidate and related drugs in human volunteers, patients with ADHD, and experimental animals. In: Solanto M, Arnsten A, Castellanos F, editors. Stimulant drugs and ADHD basic and clinical neuroscience. New York: Oxford University Press; 2001. p. 303-31.

8. Spencer RC, Klein RM, Berridge CW. Psychostimulants act within the prefrontal cortex to improve cognitive function. Biol Psychiatry. 2012;72:221-7.

9. Wang $M$, Ramos BP, Paspalas CD, Shu Y, Simen A, Duque A, et al. Alpha2Aadrenoceptors strengthen working memory networks by inhibiting CAMP-HCN channel signaling in prefrontal cortex. Cell. 2007;129:397-410.

10. Tanila H, Rama $P$, Carlson $S$. The effects of prefrontal intracortical microinjections of an alpha-2 agonist, alpha-2 antagonist and lidocaine on the delayed alternation performance of aged rats. Brain Res Bull. 1996;40:117-9.

11. Denney C, Rappoport M. Cognitive pharmacology of stimulants in children with ADHD. In: Solanto M, Arnsten A, Castellanos F, editors. Stimulant drugs and ADHD: basic and clinical neuroscience. Oxford: Oxford University Press; 2001. p. 283-302.

12. Pievsky MA, McGrath RE. Neurocognitive effects of methylphenidate in adults with attention-deficit/hyperactivity disorder: a meta-analysis. Neurosci Biobehav Rev. 2018;90:447-55.

13. Sprague RL, Sleator EK. Methylphenidate in hyperkinetic children: differences in dose effects on learning and social behavior. Science. 1977;198:1274-6.

14. Tannock R, Schachar RJ, Carr RP, Logan GD. Dose-response effects of methylphenidate on academic performance and overt behavior in hyperactive children. Pediatrics. 1989;84:648-57.

15. Voorn P, Vanderschuren $\sqcup$, Groenewegen HJ, Robbins TW, Pennartz CM. Putting a spin on the dorsal-ventral divide of the striatum. Trends Neurosci. 2004;27:468-74.

16. Sesack SR, Deutch AY, Roth RH, Bunney BS. Topographical organization of the efferent projections of the medial prefrontal cortex in the rat: an anterograde tract-tracing study with Phaseolus vulgaris leucoagglutinin. J Comp Neurol. 1989;290:213-42.

17. Ragozzino ME. The contribution of the medial prefrontal cortex, orbitofrontal cortex, and dorsomedial striatum to behavioral flexibility. Ann NY Acad Sci. 2007;1121:355-75

18. Seamans JK, Phillips AG. Selective memory impairments produced by transient lidocaine-induced lesions of the nucleus accumbens in rats. Behav Neurosci. 1994;108:456-68.

19. Kuczenski R, Segal DS. Effects of methylphenidate on extracellular dopamine, serotonin, and norepinephrine: comparison with amphetamine. J Neurochem. 1997;68:2032-7.

20. Kodama T, Kojima T, Honda Y, Hosokawa T, Tsutsui K, Watanabe M. Oral administration of methylphenidate (Ritalin) affects dopamine release differentially between the prefrontal cortex and striatum: a microdialysis study in the monkey. J Neurosci. 2017;37:2387-94.

21. Vijayraghavan S, Wang M, Birnbaum SG, Williams GV, Arnsten AF. Inverted-U dopamine D1 receptor actions on prefrontal neurons engaged in working memory. Nat Neurosci. 2007;10:376-84.

22. Zahrt J, Taylor JR, Mathew RG, Arnsten AF. Supranormal stimulation of D1 dopamine receptors in the rodent prefrontal cortex impairs spatial working memory performance. J Neurosci. 1997;17:8528-35.

23. Arnsten AFT, Pliszka SR. Catecholamine influences on prefrontal cortical function: relevance to treatment of attention deficit/hyperactivity disorder and related disorders. Pharmacol Biochem Behav. 2011;99:211-6.

24. Lapiz MD, Morilak DA. Noradrenergic modulation of cognitive function in rat medial prefrontal cortex as measured by attentional set shifting capability. Neuroscience. 2006;137:1039-49.

25. Aston-Jones G, Rajkowski J, Cohen J. Locus coeruleus and regulation of behavioral flexibility and attention. Prog Brain Res. 2000;126:165-82 [cited 21 August 2015]. http://www.sciencedirect.com/science/article/pii/S0079612300260135

26. Aston-Jones G, Rajkowski J, Cohen J. Role of locus coeruleus in attention and behavioral flexibility. Biol Psychiatry. 1999;46:1309-20.

27. Gescheider GA. Psychophysics: the fundamentals [Internet]. Psychology Press; 2013 [cited 26 Oct 2018]. https://www.taylorfrancis.com/books/9781134801220

28. Devilbiss D, Jenison R, Berridge C. Stress-induced impairment of a working memory task: role of spiking rate and spiking history predicted discharge. PLoS Comput Biol. 2012;8:e1002681. 
29. Devilbiss DM, Spencer RC, Berridge CW. Stress degrades prefrontal cortex neuronal coding of goal-directed behavior. Cereb Cortex. 2016;27:2970-83.

30. Granon S, Passetti F, Thomas KL, Dalley JW, Everitt BJ, Robbins TW. Enhanced and impaired attentional performance after infusion of D1 dopaminergic receptor agents into rat prefrontal cortex. J Neurosci. 2000;20:1208-15.

31. Bondi CO, Jett JD, Morilak DA. Beneficial effects of desipramine on cognitive function of chronically stressed rats are mediated by alpha1-adrenergic receptors in medial prefrontal cortex. Prog Neuropsychopharmacol Biol Psychiatry. 2010;34:913-23.

32. Arnsten AF, Mathew R, Ubriani R, Taylor JR, Li BM. Alpha-1 noradrenergic receptor stimulation impairs prefrontal cortical cognitive function. Biol Psychiatry. 1999;45:26-31.

33. Rubia K, Halari R, Cubillo A, Smith AB, Mohammad A-M, Brammer $M$, et al. Methylphenidate normalizes fronto-striatal underactivation during interference inhibition in medication-naive boys with attention-deficit hyperactivity disorder. Neuropsychopharmacology. 2011;36:1575-86.

34. Vaidya CJ, Austin G, Kirkorian G, Ridlehuber HW, Desmond JE, Glover GH, et al. Selective effects of methylphenidate in attention deficit hyperactivity disorder: a functional magnetic resonance study. Proc Natl Acad Sci USA. 1998;95:14494-9.

35. Tannock R, Schachar R, Logan G. Methylphenidate and cognitive flexibility: dissociated dose effects in hyperactive children. J Abnorm Child Psychol. 1995;23:235-66.

36. Mao ZM, Arnsten AF, Li BM. Local infusion of an alpha-1 adrenergic agonist into the prefrontal cortex impairs spatial working memory performance in monkeys. Biol Psychiatry. 1999;46:1259-65.

37. Cubillo A, Halari R, Smith A, Taylor E, Rubia K. A review of fronto-striatal and fronto-cortical brain abnormalities in children and adults with attention deficit hyperactivity disorder (ADHD) and new evidence for dysfunction in adults with ADHD during motivation and attention. Cortex. 2012;48: 194-215.

38. Casey BJ, Epstein JN, Buhle J, Liston C, Davidson MC, Tonev ST, et al. Frontostriatal connectivity and its role in cognitive control in parent-child dyads with ADHD. Am J Psychiatry. 2007;164:1729-36.
39. Liston C, Malter Cohen M, Teslovich T, Levenson D, Casey BJ. Atypical prefrontal connectivity in attention-deficit/hyperactivity disorder: pathway to disease or pathological end point? Biol Psychiatry. 2011;69:1168-77.

40. Vertes RP. Differential projections of the infralimbic and prelimbic cortex in the rat. Synapse. 2004;51:32-58.

41. Vertes RP. Interactions among the medial prefrontal cortex, hippocampus and midline thalamus in emotional and cognitive processing in the rat. Neuroscience. 2006;142:1-20.

42. Gamo NJ, Wang M, Arnsten AFT. Methylphenidate and atomoxetine enhance prefrontal function through a2-adrenergic and dopamine D1 receptors. J Am Acad Child Adolesc Psychiatry. 2010;49:1011-23.

43. Kolar D, Keller A, Golfinopoulos M, Cumyn L, Syer C, Hechtman L. Treatment of adults with attention-deficit/hyperactivity disorder. Neuropsychiatr Dis Treat. 2008;4:389-403.

44. Robbins TW, Arnsten AFT. The neuropsychopharmacology of fronto-executive function: monoaminergic modulation. Annu Rev Neurosci. 2009;32:267-87.

45. Arnsten AF, Li BM. Neurobiology of executive functions: catecholamine influences on prefrontal cortical functions. Biol Psychiatry. 2005;57:1377-84.

46. Arnsten AF, Steere JC, Hunt RD. The contribution of alpha 2-noradrenergic mechanisms of prefrontal cortical cognitive function. Potential significance for attention-deficit hyperactivity disorder. Arch Gen Psychiatry. 1996;53:448-55.

47. Arnsten AFT. Catecholamine and second messenger influences on prefrontal cortical networks of "representational knowledge": a rational bridge between genetics and the symptoms of mental illness. Cereb Cortex. 2007;17(Suppl 1): i6-15.

48. Arnsten AF. Through the looking glass: differential noradenergic modulation of prefrontal cortical function. Neural Plast. 2000;7:133-46.

49. Hupalo S, Berridge CW. Working memory impairing actions of corticotropinreleasing factor (CRF) neurotransmission in the prefrontal cortex. Neuropsychopharmacology. 2016;41:2733-40.

50. Diamond A. Attention-deficit disorder (attention-deficit/hyperactivity disorder without hyperactivity): a neurobiologically and behaviorally distinct disorder from attention-deficit/hyperactivity disorder (with hyperactivity). Dev Psychopathol. 2005;17:807-25. 